\title{
The influence of oral health and psycho-social well-being on clinical outcomes in Behçet's disease
}

\author{
Amal Senusi $^{1}$ - Stephen Higgins ${ }^{2} \cdot$ Farida Fortune $^{1}$
}

Received: 20 June 2018 / Accepted: 31 July 2018 / Published online: 27 August 2018

(c) The Author(s) 2018

\begin{abstract}
This study was designed to investigate the association of oral ulceration and oral health factors, together with psycho-social well-being in Behçet's disease (BD), and to clarify the importance of psycho-social support of patients in the overall management of BD. The study comprised of a cohort of $146 \mathrm{BD}$ patients (mean age $\pm \mathrm{SD}=39.65 \pm 13.20$ ) and 20 recurrent aphthous stomatitis (RAS) patients (mean age $\pm \mathrm{SD}=42.32 \pm 11.32$ ). Oral ulcer severity score (OUSS), Behçet's disease current activities form (BDCAF), hospital anxiety and depression scale (HADS), and the work and social adjustment scale (WSAS) were investigated. Oral health risk factors were also included. The analysis of variance, regression, and factor analysis were used to scrutinise the data. Almost $73 \%$ of patients were at high caries risk in BD and RAS groups. Thirty-nine percent of BD and forty percent of RAS had a score of BPE3 (probing depth 3.5-5.5 mm). Regression analysis revealed that OUSS and WSAS had a positive impact to increase the BDCAF score in BD patients $(\beta=0.395, P=0.001 ; \beta=0.240$, $P=0.019$ ), respectively. Dental health, periodontal health, anxiety, depression, and WSAS variables had strong loadings by factor analysis based on gender and at the time of present and absent of oral ulceration. The main oral ulcer characteristics that had significant influences on the total of oral health quality of life by $68.6 \%$ were: size, duration, ulcer-free period, and pain. The results highlighted the significant influence of oral ulceration, patients' oral health, diet, and psycho-social wellbeing as multi-factorial causes on increasing disease activity in BD patients.
\end{abstract}

Keywords Oral ulcer severity score $\cdot$ Periodontal risk factors · Quality of life $\cdot$ Disease activity $\cdot$ Psycho-social well-being

Electronic supplementary material The online version of this article (https://doi.org/10.1007/s00296-018-4117-y) contains supplementary material, which is available to authorized users.

Farida Fortune

f.fortune@qmul.ac.uk

Amal Senusi

a.a.s.senusi@qmul.ac.uk

Stephen Higgins

Steve.Higgins@bartshealth.nhs.uk

1 Centre for Immunobiology and Regenerative Medicine, Institute of Dentistry, Barts and the London School of Medicine and Dentistry, Blizard Institute, Queen Mary University of London, 4 Newark Street, London E1 2AT, UK

2 Behçet's Centre of Excellence, Royal London Hospital, London, UK

\section{Introduction}

Behçet's disease (BD) is a chronic, multi-system vasculitic disease. The aetiopathogenesis of BD remains unknown, and more recently, BD has been regarded as an auto-inflammatory condition. It is characterised with relapsing episodes of oro-genital ulcers accompanied by cutaneous lesions (pseudo folliculitis and erythema nodosum-like lesion), ocular symptoms (anterior uveitis, cataract, glaucoma, posterior segment involvement, and retinitis), arthropathy, vascular (arterial and venous vasculitis, and thrombosis), central nervous system (headache and meningoencephalitis), and gastrointestinal involvements (mucosal ulceration and perforation) $[1,2]$. It may cause a significant morbidity such as blindness, loss of balance, deep venous thrombosis, and neurological problems [3]. In addition, fatigue and disturbance of sleep patterns are common in BD patients. The incidence is almost equal in males and females, although males often present with more severe symptoms [4]. Furthermore, the complex pattern of symptoms in adults with BD can have a 
negative effect on their psychological, physical, and social well-being, and can cause varying degrees of limitation of activity in everyday life [5].

\section{Oral ulceration and oral health in BD}

The presence of painful recurrent oral ulceration is the first and the most common manifestation of BD which occurs in $80-100 \%$ of the patients, The ulcers may present in three different morphological patterns: minor ulcers, major ulcers, and herpetiform ulcers [6]. The cause of oral ulcers in BD is not fully understood. However, the oral ulceration in BD may be triggered by an abnormal immune response to an autoantigen. In addition, the clinical observations of BD suggest a potential role for antigens, especially organism, e.g., Herpes Simplex virus or Streptococcus Spp. in the pathogenesis. These infective agents may work through molecular mimicry, attracting inflammatory cells into the ulcerative area, and other cites of disease activity [7, 8]. Oral ulcers may also occur after local trauma causing during dental treatment [7,9]. Other organisms such as Candidiasis are not uncommon in BD patients who are treated with immunosuppression [10]. Furthermore, the oral cytokines and immunomodulatory medication may also produce changes in the oral microflora in BD patients and causing increased pathogenicity $[11,12]$. There is evidence that restoring the balance of the microbial community particularly in patients' oral cavity was promoted increased healing of oral ulcers [13].

The previous literature suggests that, during the diseases activity, patients usually have (1) reduced oral hygiene, (2) high plaque accumulation, (3) decrease their tooth brushing activity, and (4) increase their intake of sugary drinks and soft foods which alters the oral $\mathrm{pH}$ and increased cytokines activity in saliva and gingival crevicular fluid and cause tooth decay and increase the number of extracted teeth due to multiple carious lesions $[14,15]$. These factors are involved in the progression of the periodontal disease and can act to trigger their immune system and exacerbate the oral and systemic symptoms [16]. In addition, other studies have demonstrated the relationship of oral health and periodontal diseases in patients with BD compared to healthy participants [14, 17-19]. This suggests that improving the oral care of BD patients by plaque control methods: dental brushing, dental flossing, and regular dental examination may positively affect the course of the disease and prognosis [20] and may improve BD patients' quality of life (QoL).

The frequent episodes of oral ulceration in $\mathrm{BD}$ patients subsequently cause mucosal changes including obliterative scarring. Our clinical observations have been that oral scars are seen in BD patients with major or herpetiform ulceration and particularly when the ulcers recurrent in the same location. Classically, BD patients who have ulcers in the oropharynx and oesophagus may develop severe scarring which may lead to oropharyngeal stenosis [21]. These scars can cause functional problems and may interfere with both swallowing and ultimately breathing. In addition, the changes have a negative impact on the patients' QoL [22]. Besides, the oral health impact profile-14 (OHIP-14) questionnaire suggests that oral health problems in BD can alter the patients physical appearance leading to a reduction in QoL by impairing physical-social functioning and selfrespect [23-25].

\section{Psycho neuro-immunology}

Behavioural-neural-endocrine-immune system interactions suggest that psychological stress and negative emotions are related to an increase in the level of pro-inflammatory cytokines, e.g., interleukin (IL)- $1 \beta$ and IL-6, and predict illness and mortality [26, 27]. The increased levels of pro-inflammatory cytokines activate the hypothalamus and subsequently lead to "sickness behaviour". This can disturb sleep, eating behaviour, and mood states, with the feelings of discomfort and low energy [28].

The link between long-term medical conditions, health, and well-being has been a focus of concern for service commissioners for several years [29]. Currently, there is interest in assessing the impact of oral health status on QoL and in assessing the impact of oral healthcare. Clinical oral health status measures have provided some insight into the physical, social, and psychological consequences of oral health.

Psychological stress and anxiety is known to have a significant role as a trigger or modifying factor in the onset and recurrence of recurrent aphthous stomatitis (RAS) [30-32], burning mouth syndrome (BMS) [33], and lichen planus (LP) lesions [34]. In response to stress, the alteration of hypothalamic-pituitary-adrenal axis (HPA) can lead to elevation of cortisol levels and a consequential activation of the immune system with an increased number of leukocytes at the sites of inflammation [35].

The focus of this research was to study the factors in the patients' protocols that might impact on the BD patients' outcomes including oral health, disease activity and oral health-related quality of life. These clinical outcomes might then assist in providing a more comprehensive approach to the patients' overall management.

In this study, we have:

1. investigated the role of oral health factors, psycho-social well-being, oral ulcer severity, and disease activity in the BD patients based on the patients' gender and oral ulceration (present/absent) episodes;

2. assessed the oral ulcer severity and OHQoL in RAS and BD by using the OUSS a new validated assessment tool; 
3. explored the need of including oral health management and psycho-social support as a part of treatment guidelines of BD patients.

\section{Methods}

The study was a year-long prospective study using a convenience sampling technique and was performed at Behçet's centre (BD patients) and Oral Medicine Clinic (RAS patients) at the Royal London Hospital. BD and RAS participants were consented and included in this study which is a part of the City Research Ethical Committee (COREC) approved study "Immune-regulation at the mucosal barrier 14 November 2006" (P/03/122).

The eligibility criteria for inclusion patients were: for the $\mathrm{BD}$, cohort was those patients diagnosed with BD according to International Study Group (ISG) 1990 criteria [36, 37]. The RAS cohort was those presenting with typical aphthous ulceration but no underlying haematinic deficiency or systemic disease. In both groups BD and RAS, all patients were between 18 and 65 years old. All females who were pregnant or lactating were excluded from both the BD and RAS group. The sample size was calculated using a specific formula that performed by Singh and Masuku [38]. The optimal number required in this study was 130 patients, and in this study, a large cohort of 264 BD patients had the oral health assessments: periodontal indices, periodontal risk factors, and dental caries index, OUSS, and OHQoL data, 157 females (mean age $\pm \mathrm{SD}=39.83 \pm 13.42$ ) and 107 males (mean age $\pm \mathrm{SD}=39.98 \pm 11.95$ ). In addition, $146 \mathrm{BD}$ patients had all the oral health assessment plus to their psychological and social data (mean age $\pm \mathrm{SD}=39.65 \pm 13.20$ ). Twenty RAS patients (mean age $\pm \mathrm{SD}=42.32 \pm 11.32$ ) from the oral medicine clinic were also included.

\section{Outcome measures}

\section{Oral ulcer severity score (OUSS) form}

The OUSS tool validated for BD and was provided to assess oral ulcer severity [39, 40]. The six OUSS characteristics: number, size, duration of ulcers, ulcer-free period, pain, and site of ulcers were recorded. The OUSS form used included evidence of scarring, to evaluate the severity of the oral ulceration in patients' cohort; in addition, the effect of oral ulceration on normal life activity was investigated. The patients' medication was also recorded for monitoring the efficacy of the treatment protocols for each patient. To complete the OUSS form, the ulcers' characteristics were converted into numerical values, to assess the oral ulcers in BD and RAS patients for the last 4 weeks (Table S1).

\section{Dental and periodontal examination}

The dental and periodontal examination was carried out by the researcher on the day of clinical assessment of the patients. Decayed missing and filled teeth (DMFT) index was used for presenting the epidemiological data of the caries experience in BD and RAS cohort. When a carious lesion and a restoration are present, the tooth was recorded as a (D). When a tooth has been extracted due to caries, it was recorded as a $(\mathrm{M})$. When a permanent or temporary filling was present, or when a filling was defective but not decayed, this was counted as a $(F)$. The teeth not counted were: un-erupted teeth, congenitally missing teeth or supernumerary teeth, teeth removed, restored for reasons other than dental caries, third molars, and primary teeth retained in the permanent dentition.

Basic periodontal examination (BPE) was provided as a rapid-screening tool, and it was used to indicate the level of further examination needed and provide the basic guides on treatment need. BPE scores were given to each sextant, from 0 to 4 , and * indicates dental furcation involvement [41] (Figure S1). World health organisation probe (WHO) was used for BPE. This has a "ball end" of $0.5 \mathrm{~mm}$ in diameter, and a black band from 3.5 to $5.5 \mathrm{~mm}$. Light probing force was indicated (20-25 g). Sulcus bleeding index (BI) assessed the early sign of gingivitis [42], and plaque index (PI) assessed the thickness of plaque at the gingival area were applied for all the patients [43]. The patients were asked closed-ended questions answered with "Yes" or "No"; these included (1) patient's gingival swelling or bleeding and family members swelling or bleeding; (2) smoking; (3) balanced diet; (4) medical problems, e.g., as diabetes, cardiovascular diseases and other comorbidities; (5) stress, and (6) oral hygiene habits (the frequency of brushing teeth, flossing, and cleaning tongue) were taken from all the patients; (7) plaque retention factors such as: supra-gingival calculus, sub-gingival calculus, and poor restoration margin were investigated by researcher.

\section{Behçet's disease current activity form (BDCAF)}

The BDCAF form is a well-established tool for the assessment of BD activity in the clinic [44], which scores the history of clinical features on a $0-12$ scale based on the number of symptoms which present over the preceding 4 weeks, prior to the day of assessment: these include headache, oral ulcer, genital ulcer, skin lesions, ocular 
symptoms, joint involvement, blood vessel involvement, and gastrointestinal and CNS complications.

\section{Psychological and social well-being scales}

Two measurement scales were used to evaluate the psychological and social well-being of patients; (1) hospital anxiety and depression scale (HADS), developed by Zigmond and Snaith [45], is a valid and convenient selfrating instrument for anxiety and depression in patients with somatic and psychological disorders. The questionnaire features seven questions relating to anxiety and seven relating to depression on $0-3$ scales. The HADS is divided into four ranges: normal (0-7), mild (8-10), moderate (11-15), and severe (16-21). (2) The work and social adjustment scale (WSAS) is another valid and reliable self-report five-item questionnaire of functional impairment attributable to an identified problem or disorder on a 0-8 scale: 0 indicates no impairment at all and 8 indicates very severe impairment [46]. The range of the total score is 0-40, with lower scores indicating higher functioning. A WSAS score $>20$ suggests at least moderately severe functional impairment. Scores between 10 and 20 indicate measurable functional impairment but less severe clinical symptomatology. The HADS and WSAS questionnaires were completed by patients on the day of their clinical assessment.

\section{Statistical analysis}

The descriptive analysis was performed for mean and standard deviation values. The Pearson's coefficient test was used to interrogate the data correlation. Regression, Kruskal-Wallis test (for non-parametric data), and analysis of variance (ANOVA) tests were implemented. The relationship between the variables was assessed by multivariate regression. Principle component analysis (PCA) was performed on the study scales with the number of factors predefined by eigenvalue $\geq 1.00$ (eigenvalues are calculated and used in deciding how many factors to extract in the overall factor analysis). Varimax rotation was performed to obtain simpler factors. Rotation maximises the loading of each variable on one of the extracted factors whilst minimising the loading on all other factors. Interpretation of the PCA is based on finding which variables are most strongly correlated with each component; the correlation value $\geq 0.5$ is deemed important.

The data were analysed using the SPSS Statistics software (version 20 for Windows; IBM Corporation, New York, USA). $P$ values $<0.05$ were accepted as significant.

\section{Results}

\section{Dental and periodontal factors}

Analysis of the DMFT and caries risk demonstrated that $(106 / 146 ; 72.6 \%)$ of BD and $(15 / 20 ; 75 \%)$ of RAS patients were at high caries risk. In addition, DMFT index components were used in form of three separated factors (D, $\mathrm{M}$, and $\mathrm{F}$ factors) to be able to identify which factor/s had an impact on the total of DMFT index. Using the Kruskal-Wallis test to identify the DMF teeth in the BD group, resulted in a $P$ value of $<0.001$. However, there is no significant difference between DMF teeth in RAS patients $P=0.326$.

BPE was carried out on BD and RAS patients. In the BD cohort, a total of $(57 / 146 ; 39 \%)$ had BPE score 3 , while $(32 / 146 ; 22 \%)$ had BPE score of 4 and 7 BD patients had a BPE score of $4 *$ (indicating probing depth $>5.5 \mathrm{~mm}$ plus a furcation involvement in the sextant). However, $(41 / 146 ; 28 \%)$ patients had BPE score 2. Only three patients had BPE score 0 . Most of RAS patients $(8 / 18$; $40 \%)$ had BPE score 3 , and $(5 / 18 ; 27 \%)$ had BPE score 4. Only two patients had BPE score $4 *$. The number of patients with BPE score 0 and 1 was [BD (3 and 6), no RAS had 0 and 1 BPE score], respectively (Figure S1).

$(57 / 146 ; 39 \%)$ BD and $(7 / 20 ; 35 \%)$ RAS patients gave a history of gingival problems, and 25 and $20 \%$ have only a family history of gingival problems (gingivitis and/or periodontal diseases), respectively. BD and RAS patients with plaque retention factors were $(111 / 146 ; 76 \%)$ and $(16 / 20 ; 80 \%)$ respectively. Most of our patients in both groups BD and RAS had never smoked. Only (43/146; $29.4 \%) \mathrm{BD}$ and $(3 / 20 ; 15 \%)$ RAS were smokers with mean number of cigarette consumption $(2.5 \pm 6.42 /$ day $)$ and $(3.2 \pm 7.22$ day), respectively. Around $45 \%$ of our patients presented with only BD or RAS had underlying comorbidities. Six patients had BD and diabetes, 7 BD patients had cardiovascular disease, and 22 patients had BD with other medical conditions. Depression, anxiety, or other psychological and social problems were diagnosed in $(54 / 146 ; 37 \%) \mathrm{BD}$ and $(7 / 20 ; 35 \%)$ in RAS patients. For most of our BD and RAS cohort, the diet was balanced and included all types of nutrition (according to the diet form and clinician decision). In terms of oral hygiene habits, most of the patients brushed their teeth at least twice/day and did irregular flossing and tongue brushing (Table S2).

The distribution of BDCAF scores (overall BDCAF score $=12)$ in BD patients was as follows: $(6 / 146 ; 4 \%)$ patients had a score of $0,(9 / 146 ; 6 \%)$ patients had a score of 1 , and $(11 / 146 ; 7.5 \%)$ patients scored $2 .(20 / 146 ; 13.7 \%)$ patients scored 3 and 5 each, $(24 / 146 ; 16.5 \%)$ patients had a score of $4,(21 / 146 ; 14.4 \%)$ patients scored $6,(12 / 146$; 
$8 \%)$, and $(15 / 146 ; 10 \%)$ patients had a score of 7 and a score of 8 respectively. $(7 / 146 ; 5 \%)$ patients scored 9 , and only one patient scored 10 .

Multivariate regression analysis and matrix correlation analysis demonstrated a moderate correlation between BPE with BI, PI, and with DMFT in BD $[(R=0.617$; $P<0.001),(R=0.408 ; P<0.001)$, and $(R=0.549$; $P<0.001)]$. However, RAS group had a strong correlation between BPE with $\mathrm{BI}$ and with PI $(R=0.74$; $P<0.001, R=0.78 ; P<0.001)$. There was a strong correlation between $\mathrm{PI}$ and $\mathrm{BI}$ in RAS $(R=0.76 ; P<0.001)$ and moderate correlation in $\mathrm{BD}(R=0.59 ; P<0.001)$. There was a correlation between PI score and OUSS in both groups. The anxiety (mean $\pm \mathrm{SD}=9.16 \pm 5$ ), depression (mean $\pm \mathrm{SD}=6.79 \pm 4.69)$, and the WSAS level (mean $\pm \mathrm{SD}=19.75 \pm 9.65$ ) were high in BD patients compared with RAS patients. The oral ulcer severity and psychological and social well-being were observed to be risk factors to increase the systemic activity in BD patients $\left(R^{2}=0.23, P<0.001\right)$.

\section{The effect of gender on the oral health, and psychological and social well-being on BD}

Principal component analysis (PCA) was used to analyse the effect of gender on oral health, disease activity, and psychological and social well-being. In these data, the largest eigenvalue, that is the eigenvalue of the first principal component, is $(\mathrm{F} 1=3.61)$ in females group and $(\mathrm{M} 1=4.22)$ in males group, and, therefore, explains 21.24 and $24.82 \%$ of the variation in the original data of females and males' groups, respectively. Similarly, the eigenvalues of the second and third components were (15.33 and 10.24\%) and (15.35 and $13.10 \%$ ), respectively. The first three factors: F1, F2, and $\mathrm{F} 3$ together, therefore, account for $47.10 \%$ in women and $53.21 \%$ in men (M1, M2, and M3) of the variance in the original data. This information is summarized in Table 1 which also shows PC1 of BD females; under the heading, 'F1' has positive loadings on those variables [age, DMFT, number of filled teeth, BPE, and BI]. The PC2 under the heading ' $\mathrm{F} 2$ ' has positive loadings on the [BPE, BI, PI, and anxiety]. The third component under the heading ' $\mathrm{F} 3$ '
Table 1 Oral health, and psychological and social wellbeing of females and males BD patients

\begin{tabular}{|c|c|c|c|c|c|c|}
\hline \multicolumn{7}{|c|}{ Principle component analysis results ${ }^{\mathrm{a}}$} \\
\hline & \multicolumn{6}{|c|}{ Component } \\
\hline & $\mathrm{F} 1$ & $\mathrm{~F} 2$ & F3 & M1 & M2 & M3 \\
\hline Age range & 0.691 & -0.093 & 0.114 & 0.124 & 0.630 & -0.105 \\
\hline DMFT index & 0.925 & 0.216 & -0.009 & 0.944 & 0.209 & 0.006 \\
\hline Decayed teeth & -0.139 & -0.043 & 0.094 & 0.254 & 0.055 & 0.271 \\
\hline Missing teeth & 0.337 & 0.301 & 0.145 & 0.717 & 0.323 & -0.152 \\
\hline Filled teeth & 0.857 & 0.080 & -0.114 & 0.789 & 0.053 & -0.065 \\
\hline BPE index & 0.530 & 0.636 & 0.132 & 0.521 & 0.633 & -0.183 \\
\hline Bleeding index & 0.501 & 0.755 & -0.119 & 0.334 & 0.771 & -0.010 \\
\hline Plaque index & 0.002 & 0.786 & 0.141 & -0.005 & 0.818 & 0.115 \\
\hline OUSS & -0.314 & 0.272 & 0.608 & 0.038 & 0.071 & 0.113 \\
\hline Smoking & 0.061 & 0.078 & -0.376 & 0.183 & 0.307 & -0.203 \\
\hline Plaque retention factors & 0.026 & -0.049 & 0.000 & -0.193 & -0.083 & -0.019 \\
\hline Medical factors & -0.198 & -0.187 & -0.064 & -0.083 & -0.368 & -0.226 \\
\hline Diet & 0.013 & 0.155 & -0.081 & -0.117 & 0.280 & -0.588 \\
\hline BDCAF & -0.013 & 0.186 & 0.569 & 0.286 & -0.035 & 0.334 \\
\hline Anxiety & -0.184 & 0.674 & 0.264 & -0.222 & -0.150 & 0.829 \\
\hline Depression & 0.078 & 0.418 & 0.593 & -0.150 & 0.223 & 0.773 \\
\hline WSAS & 0.133 & -0.060 & 0.824 & 0.116 & -0.037 & 0.722 \\
\hline Eigenvalues & 3.61 & 2.61 & 1.75 & 4.22 & 2.61 & 2.22 \\
\hline Explained variance & $21.24 \%$ & $15.33 \%$ & $10.24 \%$ & $24.82 \%$ & $15.35 \%$ & $13.10 \%$ \\
\hline Cumulative variance & $21.53 \%$ & $36.86 \%$ & $47.10 \%$ & $24.82 \%$ & $40.17 \%$ & $53.21 \%$ \\
\hline
\end{tabular}

Bold indicates factor loading $\geq 0.50$ in the distribution of the oral health and psychological items Extraction method: principal component analysis

Rotation method: varimax with kaiser normalization

$B P E$ basic periodontal examination, BDCAF Behçet's disease current activities form, DMFT decayed missing and filled teeth, OUSS oral ulcer severity score, WSAS the work and social adjustment scale (WSAS)

${ }^{\mathrm{a}}$ Only cases for which Genders of $\mathrm{BD}=$ Female $(\mathrm{F})$ and Male $(\mathrm{M})$ were used in the analysis phase 
increased with increasing OUSS, BDCAF, depression, and WSAS, respectively. However, the PC1 in BD males under heading 'M1' has the same females' variables; 'F1' except $\mathrm{BI}$ variable was less importance in males group, and the age range was important in males group in PC2 ' $\mathrm{M} 2$ '. The third component of BD males under the heading 'M3' soared with increasing the [anxiety, depression, and WSAS] and decreasing strongly with value of diet variable. This component 'M3' might be viewed that the healthy and balanced diet is a crucial factor in males BD group.

It may be stated that, based on the DMFT correlation of 0.925 and 0.944 in the first components of females and males, respectively, these F1 and M1 are primarily a measure of the dental health. Whereas, F2 and M2 are measures for dental plaque on the correlation of 0.786 and 0.818 . Finally, the prominent measure in F3 was WSAS and that in M3 was the anxiety. In summary, the key findings that DMFT and dental plaque were the main variables in both females and males. Moreover, F3 was primarily a measure for the work and social status. However, M3 was a measure for anxiety.

\section{The effect of oral ulceration on oral health, and psychological and social well-being in BD}

The first three factors (PC1, PC2, and PC3) represent BD group with no oral ulceration (absence of oral ulceration), under the heading: AU1, AU2, and AU3 (eigenvalue $\geq 1.00$ ) of accounted for $58.32 \%$ of the variance in the original data, while, in the group of oral ulceration (presence of oral ulceration), under the heading: PU1, PU2, and PU3, the cumulative variance value was $43.79 \%$. The PC1 under heading 'PU1' of BD patients with oral ulceration was elevated (positive loading) with the following contributing variables (age, DMFT, number of filled and missing teeth, and BPE). The second component under the heading 'PU2' had positive loadings on (gender, BPE, BI, and PI). PC 3 in BD group had strong positive values (BDCAF, anxiety, depression, and WSAS). However, the absence of oral ulceration in (PC1) of BD patients 'AU1' increased with fewer the number of decayed teeth and comorbidities such as diabetes and cardiovascular diseases and other medical problems, and 'AU1' increased with increasing the (age, number of filled teeth, and DMFT). The component 'AU2' increased with positive loading of the (BPE, BI, PI, and diet). The psychological and social well-being variables had positive loadings values with AU3 and PU3 components (Table 2). In summary, the first principle component of the absence of oral ulceration group (AU1) increased by decreasing the number of decayed teeth and increasing the number of filled teeth with the control being other medical conditions. AU2 is a primarily measure for dental plaque and diet. PU1 is a primarily measure for the DMFT index and PU2 for periodontal health. AU3 and PU3 are primarily a measure for the psychology and social well-being.

\section{Oral health in BD and RAS patients}

Table 3 displays that $\mathrm{PC} 1$ under heading 'BD1' BD patients had positive loadings on [age, DMFT, BPE, and number of filled teeth]. The second principal component under the heading 'BD2' and the first principle component of RAS cohort under heading 'RAS1' had strong loadings on those variables (BPE, BI, and PI). 'BD3' with high values would tend to have a lot of plaque retention factors, in terms of supra-gingival calculus, sub-gingival calculus, and poor restoration margins, and 'BD3' component increased with increasing of medical problems. In summary, the RAS1 had a strong correlation with bleeding index of 0.955 that this principal component is primarily a measure of the periodontal health. RAS2 and RAS3 are responsible for dental and periodontal health. BD1 is primarily a measure of the dental and periodontal health. BD2 and BD3 were primary measures for the dental plaque and medical conditions, respectively.

\section{The association of OUSS with BDCAF score in BD patients}

The analysis of regression test displayed that the study model (Dependent variable: BDCAF scores, and independent variable: overall OUSS) was significant $(R=0.395$; $\left.R^{2}=0.156 ; P=0.001\right)$ (Table 4$)$, and it indicated that the overall OUSS was responsible for more than $39.5 \%$ of the variance to increase the BDCAF score of BD patients.

\section{The association of psychology and social well-being with BDCAF score in BD patients}

Table 5 indicates that the model of BDCAF scores as a dependent variable and the independent variables being anxiety scores, depression scores, and WSAS scores was associated significantly $\left(R=0.373 ; R^{2}=0.139 ; P=0.002\right)$. This also showed that the main influence to increase the BDCAF scores of BD patients was from the WSAS variable by $24 \%$ of the variance $(\beta=0.24 ; P=0.019)$. In addition, there was no significant impact of medication on the BD patients' psycho-social status.

\section{The effect of oral ulcer characteristics on OHQOL in BD}

The multivariate linear regression analysis (Table 6a) indicated that ulcer characteristics had an influence on the overall of OHQoL $\left(R^{2}: 0.686 ; P<0.001\right)$. The beta values 
Table 2 Oral health, and psychological and social wellbeing of BD groups at the time of absence and presence of oral ulceration

\begin{tabular}{|c|c|c|c|c|c|c|}
\hline \multicolumn{7}{|c|}{ Principle component analysis results ${ }^{\mathrm{a}}$} \\
\hline & \multicolumn{6}{|c|}{ Component } \\
\hline & AU1 & AU2 & AU3 & PU1 & PU2 & PU3 \\
\hline Age range & 0.707 & 0.112 & 0.146 & 0.605 & -0.074 & 0.085 \\
\hline Gender & -0.231 & 0.169 & -0.097 & -0.235 & 0.613 & -0.186 \\
\hline DMFT index & 0.563 & 0.193 & 0.279 & 0.877 & 0.218 & -0.016 \\
\hline Decayed teeth & -0.728 & 0.040 & -0.097 & 0.006 & 0.092 & 0.062 \\
\hline Missing teeth & -0.053 & 0.261 & 0.721 & 0.498 & 0.211 & 0.060 \\
\hline Filling teeth & 0.829 & 0.038 & -0.099 & 0.811 & 0.111 & -0.083 \\
\hline BPE index & 0.358 & 0.721 & 0.174 & 0.545 & 0.653 & 0.120 \\
\hline Bleeding index & 0.375 & 0.771 & 0.118 & 0.365 & 0.796 & 0.047 \\
\hline Plaque index & 0.028 & 0.866 & 0.057 & -0.012 & 0.794 & 0.279 \\
\hline Smoking & 0.234 & 0.243 & -0.108 & 0.133 & 0.206 & -0.287 \\
\hline Plaque retention factors & -0.005 & -0.130 & -0.109 & 0.032 & 0.076 & 0.078 \\
\hline Medical factors & -0.656 & -0.418 & 0.136 & -0.021 & -0.230 & -0.005 \\
\hline Diet & -0.279 & 0.785 & -0.139 & 0.048 & 0.177 & -0.200 \\
\hline BDCAF & 0.076 & 0.040 & 0.142 & 0.168 & 0.042 & 0.534 \\
\hline Anxiety & -0.032 & -0.175 & 0.784 & -0.086 & 0.216 & 0.771 \\
\hline Depression & -0.006 & 0.217 & 0.842 & 0.034 & 0.154 & 0.825 \\
\hline WSAS & 0.404 & -0.154 & 0.693 & 0.069 & -0.132 & 0.619 \\
\hline Eigenvalues & 4.96 & 2.59 & 2.40 & 3.56 & 2.19 & 1.70 \\
\hline Explained variance & $29.16 \%$ & $15.23 \%$ & $13.94 \%$ & $20.97 \%$ & $12.88 \%$ & $9.95 \%$ \\
\hline Cumulative variance & $29.16 \%$ & $44.83 \%$ & $58.32 \%$ & $20.97 \%$ & $33.84 \%$ & $43.79 \%$ \\
\hline
\end{tabular}

Bold indicates factor loading $\geq 0.50$ in the distribution of the oral health and psychological items

Extraction method: principal component analysis

Rotation method: varimax with kaiser normalization

$B P E$ basic periodontal examination, BDCAF Behçet's disease current activities form, DMFT Decayed missing and filled teeth, OUSS Oral ulcer severity score, WSAS the work and social adjustment scale (WSAS)

${ }^{a}$ Only cases for which OUSS in $\mathrm{BD}=$ Absence of oral ulcers (AU) and Presence (PU) of oral ulcers were used in the analysis phase revealed that most of the contributions which make OHQoL worse were due to; ulcer pain, size of ulcers, ulcer-free period, and duration of ulceration $(0.509 ; 0.250 ; 0.207$; $-0.109: P<0.001,0.001,0.001$, and 0.027$)$ respectively. Eating and talking were the major factors affected by high OUSS of BD: beta values were the same for both factors (0.364, $P<0.001$ : Table 6b).

\section{Discussion}

It is widely accepted that oral health has a role to initiate or aggravate BD symptoms. This study was carried out to investigate the oral health, medical factors, psychology, and social well-being that might be associated with oral ulcer severity and disease activity.

The results showed that oral health determined by plaque retention factors, periodontal diseases, and the dental health (DMFT, particularly the number of filled teeth) were observed to be increased in both genders of BD and RAS patients. The increasing in number of filled teeth indicates that dental caries rates were higher in BD patients which also suggests that our patients' cohorts were regularly attending their dental clinics where their lesions were treated. In the BD male group, the diet was an important variable and that might have an impact on the frequency and severity of oral ulceration comparing with BD female. The reasons might include the observation that the gastric intestinal tract (GIT) of males and females react differently to diet. This was apparent even when the nutritional intake is identical. In addition, sex hormones effect on the GIT microbiota composition, consequently, can trigger the immune system and cause inflammation $[47,48]$. It has been well documented that women and men have different attitudes and knowledge about nutrition and what constitutes a balanced diet, and the requirements of a healthy diet. More studies and controlled trials are needed to establish whether 
Table 3 Assessment of oral health, and psychological and social well-being of RAS and BD patients

\begin{tabular}{lllllll}
\hline Principle component analysis results & \\
& Component & & & & \\
& RAS1 & RAS2 & RAS3 & BD1 & BD2 & BD3 \\
\cline { 2 - 6 } & 0.081 & 0.097 & -0.417 & -0.268 & 0.478 & 0.257 \\
Gender & 0.361 & $\mathbf{0 . 6 1 5}$ & 0.112 & $\mathbf{0 . 6 5 2}$ & 0.076 & $\mathbf{0 . 3 0 1}$ \\
Age range & -0.047 & 0.426 & $\mathbf{0 . 8 7 8}$ & $\mathbf{0 . 9 0 7}$ & 0.238 & -0.031 \\
DMFT index & -0.052 & 0.070 & -0.008 & -0.056 & 0.068 & -0.034 \\
Decayed teeth & -0.090 & $\mathbf{0 . 9 3 2}$ & 0.140 & $\mathbf{0 . 4 9 3}$ & 0.295 & 0.174 \\
Missing teeth & 0.027 & 0.005 & $\mathbf{0 . 9 5 2}$ & $\mathbf{0 . 8 0 3}$ & 0.092 & -0.122 \\
Filling teeth & $\mathbf{0 . 8 2 2}$ & 0.450 & -0.027 & $\mathbf{0 . 5 3 4}$ & $\mathbf{0 . 6 7 2}$ & -0.128 \\
BPE index & $\mathbf{0 . 9 5 5}$ & -0.072 & -0.012 & 0.332 & $\mathbf{0 . 7 8 3}$ & -0.059 \\
Bleeding index & $\mathbf{0 . 8 7 5}$ & -0.010 & -0.010 & -0.023 & $\mathbf{0 . 8 5 6}$ & 0.002 \\
Plaque index & -0.053 & 0.016 & 0.081 & -0.197 & 0.102 & 0.106 \\
OUSS & 0.072 & 0.141 & 0.069 & 0.170 & 0.068 & 0.385 \\
Smoking & -0.351 & -0.107 & -0.037 & 0.097 & 0.022 & $\mathbf{0 . 6 8 9}$ \\
Plaque retention factors & 0.225 & -0.445 & -0.009 & -0.078 & -0.174 & $\mathbf{0 . 7 2 9}$ \\
Medical factors & -0.145 & -0.095 & -0.120 & 0.039 & 0.005 & -0.012 \\
Diet & 4.07 & 2.65 & 1.90 & 3.54 & 2.29 & 1.80 \\
Eigenvalues & $27.14 \%$ & $17.68 \%$ & $12.61 \%$ & $19.67 \%$ & $12.70 \%$ & $10.10 \%$ \\
Explained variance & $27.14 \%$ & $44.81 \%$ & $57.42 \%$ & $19.67 \%$ & $32.37 \%$ & $42.38 \%$ \\
Cumulative variance & R & & & & \\
\hline
\end{tabular}

Bold indicates factor loading $\geq 0.50$ in the distribution of the oral health and psychological items

Extraction method: principal component analysis

Rotation method: Varimax with Kaiser normalization

${ }^{a} \mathrm{RAS}$ and BD groups
Table 4 Regression analysis of OUSS with BDCAF score of BD patients

\begin{tabular}{lllll}
\hline Model summary & \multicolumn{2}{l}{$\begin{array}{l}\text { Unstandardized } \\
\text { coefficients }\end{array}$} & $\begin{array}{l}\text { Standardized } \\
\text { coefficients }\end{array}$ & $P$ value \\
\cline { 2 - 3 } & $B$ & Std. error & Beta & \\
\hline $\begin{array}{l}\text { OUSS in BD } \\
R \text { square value }=\mathbf{0 . 1 5 6}\end{array}$ & 0.066 & 0.013 & $\mathbf{0 . 3 9 5}$ & $\mathbf{0 . 0 0 1 *}$ \\
ANOVA test $; P=\mathbf{0 . 0 0 1 *}$ & & & & \\
\hline
\end{tabular}

*Bold indicates $P \leq 0.05$

${ }^{\text {a }}$ Predictor variable (independent variable): Overall OUSS of BD

${ }^{b}$ Dependent variable: BDCAF scores

the gender and diet directly affect BD and how they affect the disease presentation.

In addition, the oral health and comorbidity found in the BD and RAS groups were synergistically contributing to the high positive loading values to activate the disease. BD patients at the time of oral ulceration had those represented factors: PU1 (age, DMFT, number of filled teeth, and BPE), PU2 (BPE, PI, BI, and gender) and PU3 (BDCAF, anxiety, depression, and WSAS) were actively influencing the oral ulceration and the disease manifestations. In BD patients with active oral ulcers: the pain, size
Table 5 Regression analysis of psychology and social well-being with BDCAF score of BD patients

\begin{tabular}{|c|c|c|c|c|}
\hline \multirow[t]{2}{*}{ Model summary ${ }^{\mathrm{a}, \mathrm{b}}$} & \multicolumn{2}{|c|}{$\begin{array}{l}\text { Unstandardized } \\
\text { coefficients }\end{array}$} & \multirow{2}{*}{$\begin{array}{l}\text { Standard- } \\
\text { ized coef- } \\
\text { ficients } \\
\text { Beta }\end{array}$} & \multirow[t]{2}{*}{$P$ value } \\
\hline & $B$ & Std. error & & \\
\hline Anxiety scores in BD & 0.266 & 0.286 & 0.103 & 0.353 \\
\hline Depression scores in BD & 0.400 & 0.354 & 0.131 & 0.262 \\
\hline $\begin{array}{l}\text { WSAS scores in BD } \\
\text { patients }\end{array}$ & 0.716 & 0.301 & 0.240 & 0.019* \\
\hline \multicolumn{5}{|l|}{$R$ square value $=\mathbf{0 . 1 3 9}$} \\
\hline ANOVA test; $P=\mathbf{0 . 0 0 2} *$ & & & & \\
\hline
\end{tabular}

*Bold indicates $P \leq 0.05$

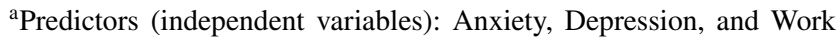
and Social Adjustment Scale (WSAS) in BD patients

${ }^{\mathrm{b}}$ Dependent Variable: BDCAF scores in BD patients

of ulcers, and duration of ulcers are the main ulcer characteristics that have an impact on OHQoL. Consequently, eating and talking are the main factors that were impaired by oral ulceration. Therefore, in our Behçet's centre, enhanced guidelines have been developed which includes topical mouth ulceration treatment (steroid mouthwash and Barts mouthwash ${ }^{\uparrow}$ ), and oral hygiene instructions are 
Table 6 Model summary and coefficients of (a) oral ulcer characteristics and overall OHQoL in BD, and (b) OUSS and OHQoL factors in BD group

\begin{tabular}{|c|c|c|c|c|}
\hline \multirow[t]{2}{*}{ Model summary ${ }^{\mathrm{a}, \mathrm{b}}$} & \multicolumn{2}{|c|}{ Unstandardized coefficients } & \multirow{2}{*}{$\begin{array}{l}\text { Standardized coefficients } \\
\text { Beta }\end{array}$} & \multirow[t]{2}{*}{$P$ value } \\
\hline & $B$ & Std. error & & \\
\hline Number of ulcers & -0.067 & 0.139 & -0.023 & 0.629 \\
\hline Size of ulcers & 0.784 & 0.152 & 0.250 & 0.001* \\
\hline Ulcers duration & -0.514 & 0.231 & -0.109 & $0.027 *$ \\
\hline Ulcer-free period & 1.232 & 0.307 & 0.207 & 0.001* \\
\hline Pain & 1.460 & 0.165 & 0.509 & 0.001* \\
\hline Site of ulcers & 0.304 & 0.170 & 0.089 & 0.076 \\
\hline \multicolumn{5}{|l|}{$R$ square value $=0.686$} \\
\hline \multicolumn{5}{|l|}{ ANOVA test; $P=\mathbf{0 . 0 0 1} *$} \\
\hline \multicolumn{5}{|l|}{ (b) } \\
\hline \multirow[t]{2}{*}{ Model summaryc,d } & \multicolumn{2}{|c|}{ Unstandardized coefficients } & Standardized coefficients & $P$ value \\
\hline & $B$ & Std. error & Beta & \\
\hline Eating in BD & 1.296 & 0.248 & 0.364 & 0.001* \\
\hline Talking in BD & 1.458 & 0.345 & 0.364 & $0.001 *$ \\
\hline Smiling in BD & 0.514 & 0.313 & 0.122 & 0.102 \\
\hline \multicolumn{5}{|l|}{$R$ square value $=0.651$} \\
\hline ANOVA test; $P=\mathbf{0 . 0 0 1} *$ & & & & \\
\hline
\end{tabular}

*Bold indicates $P \leq 0.05$

a Predictors variables: number of ulcers, size of ulcers, ulcers duration, ulcer- free period, pain, and site of ulcers

${ }^{\mathrm{b}}$ Dependent variable: Overall OHQoL of BD

${ }^{\mathrm{c}}$ Predictor variables: eating, talking, and smiling of BD patients

${ }^{\mathrm{d}}$ Dependent variable: Overall OUSS in BD

used to treat and educate our patients to follow preventive strategies, also to minimize the frequency of their disease activity and that will might lead to decrease the dose of their systemic medications (Table S3).

The strong positive loading values of psychological and social well-being variables (anxiety, depression, and WSAS) at the time of either the presences or absence of oral ulceration in the BD cohort were almost the same. This suggests that BD patients were generally at risk of mood and anxiety-related difficulties as well as experiencing impairment in their social functioning rather as a function of the presence of oral ulceration. This is not entirely surprising given that the multiple symptoms BD patients often experience together with the potential impact of a remitting-relapsing condition on their social functioning.

Psychological status has previously been identified at a higher prevalence in BD patients [49] and stress is associated with relapsing and remittance of symptoms in BD [50]. In addition, our findings showed that the WSAS status in $\mathrm{BD}$ patients had a positive impact on increasing the BDCAF scores. However, anxiety and depression did not show any association or impact on the BDCAF scores. These findings might guide us to use the patients' health questionnaire 4 (PHQ-4), patients' health questionnaire 9 (PHQ-9), and/or generalised anxiety disorder assessment (GAD-7) in any future studies instead of the hospital anxiety and depression scale (HADS), which was used in this research, as this might more accurately enable an understanding of anxiety and depression outside of the hospital setting, i.e., at home and in normal day-to-day life experience.

Interestingly, mood impacts on oral health through psychoneuroimmunological and behavioural processes. Behaviourally, low mood impacts on patients' volition to undertake everyday living and personal care activities. In oral health, depression and stress are seen to reduce pro-health behaviours such as tooth brushing, flossing, and help-seeking in people with long-term medical conditions [51], and might increase pro-disease behaviours such as smoking and drinking alcohol [52]. Psychoneuroimmunological processes influence mucosal wound healing directly [53] and psychosocial stress is associated with BD relapse generally [50]. QoL and social functioning are affected by BD disease [54] and depression [55] independently though there remains a little clarity around the interaction of BD and mood on 
social functioning in BD patients. This combined effect may be additive and form a feedback mechanism, whereby impaired social functioning exacerbates depression and possibly relapsing BD symptoms.

This study has showed the importance of oral health factors and diet in both BD and RAS patients. In addition, factor analysis demonstrated that oral health factors, medical factors, and psycho-social well-being had significant impacts on BD activity. It also highlights the validity and usefulness of OUSS as a severity monitoring tool for $\mathrm{BD}$ oral ulcers and it is recommended that it may also be useful in other Behçet's centres. In addition, including the oral hygiene instructions and using therapy mouthwashes are also highly recommended to be included in the first-line management of oral ulcers in BD patients. In addition, having a healthy balanced diet should be considered as a part of the overall management of BD patients. The PHQ-4, PHQ-9, and/or GAD-7 are important to be used in any BD future studies instead of HADS, which was used in this research and the previous studies. Stress and patients' inability to cope with the social activities and emotions might have a significant impact on BD patients. This study strongly suggests that psychological support is needed for BD patients. The Behçet's centre at Barts Health NHS Trust provides such support. Finally, this is an area of clear interest in BD patients and merits further investigation.

Acknowledgements The authors gratefully acknowledge the participation of all the BD and RAS patients, clinicians and the senior nurses; Nardos Wakjira and Sally Tillett at Behçet's centre in London of their help.

Author contributions SA contributed to the study design, collected the clinical data; in addition, SA did the data analysis, interpretation, and drafted the manuscript. HS collected the psychology and social well-being data and drafted the manuscript. FF led the project and conceived the study design and checked the data and contributed to the interpretation, and critically revised the manuscript.

\section{Compliance with ethical standards}

Conflict of interest The authors declare that they have no conflict of interests.

Ethics approval and consent to participate The study was a part of the City Research Ethical Committee (COREC) approved study "Immuneregulation at the mucosal barrier 14 November 2006" (P/03/122) and was carried out at Barts Health NHS Trust. All patients gave informed consent to the study.

Open Access This article is distributed under the terms of the Creative Commons Attribution 4.0 International License (http://creativeco mmons.org/licenses/by/4.0/), which permits unrestricted use, distribution, and reproduction in any medium, provided you give appropriate credit to the original author(s) and the source, provide a link to the Creative Commons license, and indicate if changes were made.

\section{References}

1. Chams-Davatchi C, Barikbin B (2010) Pimecrolimus versus placebo in genital aphthous ulcers of Behcet's disease: a randomized double-blind controlled trial. Int J Rheum Dis 13(3):253-258

2. Mendoza-Pinto C, García-Carrasco M, Jiménez-Hernández M, Jiménez Hernández C, Riebeling-Navarro C, Nava Zavala A et al (2010) Etiopathogenesis of Behcet's disease. Autoimmun Rev 9(4):241-245

3. Pineton de Chambrun M, Wechsler B, Geri G, Cacoub P, Saadoun D (2012) New insights into the pathogenesis of Behçet's disease. Autoimmun Rev 11(10):687-698

4. Saadoun D, Wechsler B, Desseaux K, Huong DLT, Amoura Z, Resche-Rigon M et al (2010) Mortality in Behçet's disease. Arthritis Rheum 62(9):2806-2812

5. Melikoglu MA, Melikoglu M (2010) The relationship between disease activity and depression in patients with Behcet disease and rheumatoid arthritis. Rheumatol Int 30(7):941-946

6. Bagan JV, Sanchis JM, Milian MA (1991) Recurrent aphthous stomatitis. A study of the clinical characteristics of lesions in 93 cases. J Oral Pathol Med 20(8):395-397

7. Karacayli U, Mumcu G, Simsek I, Pay S, Kose O, Erdem H et al (2009) The close association between dental and periodontal treatments and oral ulcer course in behcet's disease: a prospective clinical study. J Oral Pathol Med 38(5):410-415

8. Seoudi N, Bergmeier LA, Hagi-Pavli E (2015) The seroprevalence and salivary shedding of herpesviruses in Behçet's syndrome and recurrent aphthous stomatitis. J Oral Microbiol $7(1): 27156$

9. Yoshikawa K, Kotake S, Sasamoto Y (1991) Close association of Streptococcus sanguis and Behçet's disease. Nippon Ganka Gakkai Zasshi 95(12):1261-1267

10. Lalla RV, Latortue MC, Hong CH, Ariyawardana A, D'AmatoPalumbo S, Fischer DJ et al (2010) A systematic review of oral fungal infections in patients receiving cancer therapy. Support Care Cancer 18(8):985-992

11. Kamma JJ, Giannopoulou C (2004) Cytokine profile in gingival crevicular fluid of aggressive periodontitis: influence of smoking and stress. J Clin Periodontol 31(10):894-902

12. Bergmeier LA, Hamedi M, Hagi-Pavli E, Seoudi N, Senusi A, Ijaz H et al (2014) HSP27 as a diagnostic marker in the saliva of Behcet's disease. HSP27 as a diagnostic marker in the saliva of Behcet's disease. Immunology 143:144

13. Seoudi N, Bergmeier LA, Drobniewski F (2015) The oral mucosal and salivary microbial community of Behçet's syndrome and recurrent aphthous stomatitis. J Oral Microbiol 7(1):27150

14. Mumcu G, Ergun T, Inanc N, Fresko I, Atalay T, Hayran O et al (2004) Oral health is impaired in Behçet's disease and is associated with disease severity. Rheumatology 43(8):1028-1033

15. Yosipovitch G, Kaplan I, Calderon S, David M, Chan YH, Weinberger A (2001) Distribution of mucosal pH on the bucca, tongue, lips and palate. A study in healthy volunteers and patients with lichen planus, Behçet's disease and burning mouth syndrome. Acta Dermato-venereol 81(3):178-180

16. Seymour GJ, Gemmell E (2001) Cytokines in periodontal disease: where to from here? Acta Odontol Scand 59(3):167-173

17. Celenligil-Nazliel H, Kansu E, Ebersole JL (1999) Periodontal findings and systemic antibody responses to oral microorganisms in Behçet's disease. J Periodontol 70(12):1449-1456

18. Li X, Kolltveit KM, Tronstad L (2000) Systemic diseases caused by oral infection. Clin Microbiol Rev 13(4):547-558

19. Slavkin HC, Baum BJ (2000) Relationship of dental and oral pathology to systemic illness. JAMA 284(10):1215-1217

20. Arabaci T, Kara C, Ciçek Y (2009) Relationship between periodontal parameters and Behçet's disease and evaluation of different 
treatments for oral recurrent aphthous stomatitis. J Periodont Res 44(6):718-725

21. Keogan $M$ (2009) Clinical immunology review series: an approach to the patient with recurrent orogenital ulceration, including Behçet's syndrome. Clin Exp Immunol 156(1):1-11

22. Mehta P, Ambrose N, Haskard DO (2014) Work-related disability in Behçet's syndrome: a British case series. Clin Exp Rheumatol 32(4): 173

23. Mumcu G, Niazi S, Stewart J, Hagi-Pavli E, Gokani B, Seoudi N et al (2009) Oral health and related quality of life status in patients from UK and Turkey: a comparative study in Behcet's disease. J Oral Pathol Med 38(5):406-409

24. Mumcu G, Hayran O, Ozalp D, Inanc N, Yavuz S, Ergun T et al (2007) The assessment of oral health-related quality of life by factor analysis in patients with Behcet's disease and recurrent aphthous stomatitis. J Oral Pathol Med 36(3):147-152

25. Naito M, Suzukamo Y, Wakai K, Azechi M, Kaneko F, Nakayama $\mathrm{T}$ et al (2014) One-year period prevalence of oral aphthous ulcers and oral health-related quality of life in patients with Behçet's disease. Genet Res Int 2014:930348. https://doi. org/10.1155/2014/930348

26. Everson-Rose SA, Lewis TT (2005) Psychosocial factors and cardiovascular diseases. Annu Rev Public Health 26:469-500

27. Irwin MR (2008) Human psychoneuroimmunology: 20 years of discovery. Brain Behav Immun 22(2):129-139

28. Dantzer R, Kelley KW (2007) Twenty years of research on cytokine-induced sickness behavior. Brain Behav Immun 21(2):153-160

29. Calikoglu E, Onder M, Cosar B, Candansayar S (2001) Depression, anxiety levels and general psychological profile in Behçet's disease. Dermatology 203(3):238-240

30. Nadendla LK, Meduri V, Paramkusam G (2015) Relationship of salivary cortisol and anxiety in recurrent aphthous stomatitis. Indian J Endocrinol Metab 19(1):56

31. McCartan BE, Lamey P-J, Wallace AM (1996) Salivary cortisol and anxiety in recurrent aphthous stomatitis. J Oral Pathol Med 25(7):357-359

32. Gallo CB, Mimura MAM, Sugaya NN (2009) Psychological stress and recurrent aphthous stomatitis. Clinics 64(7):645-648

33. Amenábar JM, Pawlowski J, Hilgert JB, Hugo FN (2008) Anxiety and salivary cortisol levels in patients with burning mouth syndrome: case-control study. Oral Surg Oral Med Oral Pathol Oral Radiol Endodontol 105(4):460-465

34. Soto AM, Rojas AG, Esguep A (2003) Association between psychological disorders and the presence of Oral lichen planus, burning mouth syndrome and Recurrent aphthous stomatitis. Med Oral 9(1):1-7

35. Chiappelli F, Cajulis OS (2004) Psychobiologic views on stressrelated oral ulcers. Quintessence Int 35(3):223-227

36. O’Neill T, Rigby A, Silman A, Barnes C (1994) Validation of the International Study Group criteria for Behçet's disease. Br J Rheumatol 33(2):115-117

37. Weichsler B, Davatchi F, Mizushima Y (1990) Criteria for diagnosis of Behcet's disease. Lancet 335(8697):1078-1080
38. Singh AS, Masuku MB (2014) Sampling techniques \& determination of sample size in applied statistics research: an overview. Int J Econ 2(11): 1-22

39. Senusi A, Seoudi N, Bergmeier LA et al (2015) Genital ulcer severity score and genital health quality of life in Behçet's disease. Orphanet J Rare Dis 10(1):117

40. Tappuni A, Kovacevic T, Shirlaw P, Challacombe S (2013) Clinical assessment of disease severity in recurrent aphthous stomatitis. J Oral Pathol Med 42(8):635-641

41. George P (2014) Periodontics: BPE scores. Br Dent J 216(8):436

42. Rebelo MAB, Queiroz DAC (2011) Gingival indices: state of art. In Gingival diseases-their aetiology, prevention and treatment. InTech Open Access, London

43. Löe H (1967) The gingival index, the plaque index and the retention index systems. J Periodontol 38(6 Part II):610-616

44. Bhakta B, Brennan P, James T, Chamberlain M, Noble B, Silman A (1999) Behçet's disease: evaluation of a new instrument to measure clinical activity. Rheumatology 38(8):728-733

45. Zigmond AS, Snaith RP (1983) The hospital anxiety and depression scale. Acta Psychiatr Scand 67(6):361-370

46. Mundt JC, Marks IM, Shear MK, Greist JM (2002) The Work and Social Adjustment Scale: a simple measure of impairment in functioning. Br J Psychiatry 180(5):461-464

47. Bolnick DI, Snowberg LK, Hirsch PE, Lauber CL, Parks B, Lusis AJ et al (2014) Individual diet has sex-dependent effects on vertebrate gut microbiota. Nat Commun 29:5

48. Cordain L, Toohey L, Smith MJ (2000) Modulation of immune function by dietary lectins in rheumatoid arthritis. Br J Nutr 83(03):207-217

49. Bagheri F, Mani A, Tadayyoni A, Firozi F, Nazarinia M (2013) The prevalence of psychiatric symptoms in the patients with Behcet's disease in Shiraz, Southwest of Iran. J Mood Disord $3(1): 28$

50. Karlidag R, Unal S, Evereklioglu C, Sipahi B, Er H (2003) Stressful life events, anxiety, depression and coping mechanisms in patients with Behçet's disease. J Eur Acad Dermatol Venereol 17(6):670-675

51. Dumitrescu AL, Gârneapã L, Guzun O (2009) Anxiety, stress, depression, oral health status and behaviours in Romanian hemodialysis patients. Rom J Intern Med 47(2):161-168

52. Steptoe A, Wardle J, Pollard TM, Canaan L (1996) Stress, social support and health-related behavior: a study of smoking, alcohol consumption and physical exercise. J Psychosom Res 41(2):171-180

53. Bosch JA, Engeland CG, Cacioppo JT (2007) Depressive symptoms predict mucosal wound healing. Psychosom Med 69(7):597-605

54. Ertam I, Kitapcioglu G, Aksu K, Keser G (2009) Quality of life and its relation with disease severity in Behcet's disease. Clin Exp Rheumatol 27(2):S18

55. Hirschfeld R, Montgomery SA, Keller MB (2000) Social functioning in depression: a review. J Clin Psychiatry 61(4):268-275 